\title{
A Numerical Treatment of Melt/Solid Segregation: Size of the Eucrite Parent Body and Stability of the Terrestrial Low-Velocity Zone
}

\author{
David Walker, Edward M. Stolper, and James F. Hays \\ Hoffman Laboratory, Harvard University, Cambridge, Massachusetts 02138
}

\begin{abstract}
Crystal sinking to form cumulates and melt percolation toward segregation in magma pools can be treated with modifications of Stokes' and Darcy's laws, respectively. The velocity of crystals and melt depends, among other things, on the force of gravity $(g)$ driving the separations and the cooling time of the environment. The increase of $g$ promotes more efficient differentiation, whereas the increase of cooling rate limits the extent to which crystals and liquid can separate. The rate at which separation occurs is strongly dependent on the proportion of liquid that is present. As a result, cumulate formation is a process with a negative feedback; the more densely aggregated the crystals become, the slower the process can proceed. In contrast, melt accumulation is a process with a positive feedback; partial accumulation of melt leads to more rapid accumulation of subsequent melt. This positive feedback can cause melt accumulation to run rapidly to completion once a critical stability limit is passed. The observation of cumulates and segregated melts among the eucrite meteorites is used as a basis for calculating the $g$ (and planet size) required to perform these differentiations. The eucrite parent body was probably at least $10-100 \mathrm{~km}$ in radius. The earth's low velocity zone (LVZ) is shown to be unstable with respect to draining itself of excess melt if the melt forms an interconnecting network. A geologically persistent LVZ with a homogeneous distribution of melt can be maintained with melt fractions only on the order of $0.1 \%$ or less.
\end{abstract}

\section{INTRODUCTION}

In the presence of a gravity field, a mixture of crystals and liquid of differing density will tend to separate as a result of buoyant forces. The relative velocities of crystal and liquid leading to this separation are given by expressions which depend on the proportions of liquid and solid in the mixture. For high proportions of liquid, a modification of Stokes' law approximates the motion of particles in a fluid matrix [Shaw, 1965], while for high proportions of solid, a modification of Darcy's law approximates the motion of percolating liquid in a porous, permeable matrix [Scheidegger,, 1957]. In situations of geological interest, the extent to which crystal/melt separation occurs depends upon the balance between factors promoting greater crystal/melt mobility and those promoting heat loss, which eventually terminates motion by solidification of the system. The basic question is whether much happens before the system congeals. We explore this question by integrating expressions for melt-crystal relative velocity over the time period of a cooling episode. Since the parameters in the velocity expressions have a complex dependence on temperature, the integration is most conveniently done numerically.

Crystals and melt generally have different compositions, so their separation is a form of chemical differentiation. Using our formulation for crystal/melt separation, we can explore the range of parameters required to cause a specified amount of chemical differentiation. In this paper we use the example of the eucritic meteorites to determine what range of parameters is required to produce (1) the concentrations of cumulus pyroxene observed and (2) the segregation of eucritic melts from their source regions. With the exception of the gravitational acceleration $(g)$ of the eucrite parent body, most of the parameters required for these calculations can be estimated or are constrained by experimental petrology or petrographic or trace element studies. These calculations yield a minimum $g$ required to accomplish the diflerentiations observed in the meteorites. This value can be translated into an estimate of planet size for an assumed value of mean planet density. The calculation procedure can also be used to determine the re-

Copyright $\odot 1978$ by the American Geophysical Union. quirements for forming mechanically stable melt dispersions in the terrestrial low-velocity zone.

Consider first a horizontal plane interface separating two semi-infinite half-spaces (Figure 1). Let the lower half-space represent solidified material ('solid') and let the upper halfspace represent warmer, partially molten material ('mobile') in which crystals and liquid are free to move relative to each other. During the cooling process, the interface between solid and mobile moves upward as the mobile region solidifies. At the same time, if crystal settling occurs in the mobile region; crystals will move downward and accumulate near the rising interface. We seek to calculate the degree of crystal concentration resulting from this process.

For the related case of melt segregation, the solid region lies above the mobile zone, and the interface moves downward as solidification occurs. In this case, melt percolates upward, concentrating just below the descending solid interface. Again, the degree of concentration of the mobile phase (in this case, liquid) is desired. Mathematical formulation of the two cases is identical, and only the flow laws obeyed and the signs of the velocity vectors differ.

The degree of concentration of the mobile phase of interest is obtained by comparing the vertical spacing of two neighboring parcels of mobile material at the start $(\Delta h)$ and at the finish $\left(\Delta h^{\prime}\right)$ of the process. As the vertical spacing between parcels decreases, the concentration of the mobile phase increases. For instance, if the parcels of material decrease their vertical spacing by a factor of 2 , then $\Delta h / \Delta h^{\prime}=2$, and the concentration is doubled. The quantity desired is the concentration factor $X$, where

$$
X=\Delta h / \Delta h^{\prime}-1
$$

If no relative motion of crystals and liquid occurs, $X=0$; for the example cited, $X=1$.

The total distance traveled by each parcel is the integral of the velocity, so (1) can be rewritten to eliminate $\Delta h^{\prime}$ :

$$
X=\Delta h /\left(\int V_{2} d t-\int V_{1} d t+\Delta h\right)-1
$$

where the integrals of velocity for parcels 1 and 2 are evaluated from start to finish of the motion. The specific formulae used 


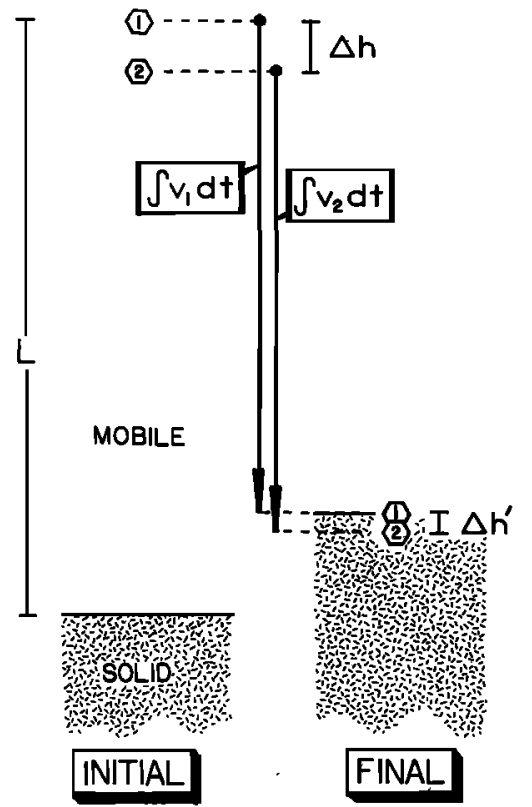

Fig. 1. Schematic of calculation procedure. Mobile parcels 1 and 2 move a distance which is the integral of velocity. Half-spaces equalize temperature by heat conduction, and congealed zone impinges on mobile zone. Concentrations achieved are computed from the initial and final spacing of the parcels $\Delta h$ and $\Delta h^{\prime}$.

for crystal sinking and melt percolation velocity contain factors that are dependent on temperature, which in turn depends on the time and the position reached by the mobile parcel during its migration. The dependence of the velocity integrals on temperature and previous concentration of mobile material is handled by numerically evaluating the integrals by computer. In the computations, three parcels of mobile material start motion at mutual spacing $\Delta h$, which is small relative to $L$, the distance the parcels start from the original mobile/congealed interface of the adjoining half spaces. Using three parcels instead of two gives a better check on numerical stability of the results. The time steps are adjusted during the numerical integration of velocity, so that the discrepancy in position of the parcel produced by using the velocity at the start of the step and the end of the step does not exceed $10^{-5} \times L$. The length of a time step is increased if this discrepancy is less than $10^{-8} \times L$. The integrations typically take about $200-500$ steps. This procedure is found to give the same numerical results to at least three significant figures as a more time-consuming integration in small fixed-time steps. For each time step, the temperature is calculated at the present position of each parcel by equation (7) of Jaeger [1957, p. 308], which is an analytic solution of the half-space heat conduction problem which includes the effect of dissipation of the latent heat of crystallization of liquid over a specified temperature interval. (Solution of equation (3) of Jaeger [1957, p. 308] for the parameter $\lambda$ giving the motion of the mobile/congealed interface, which enters into Jaeger's equation (7), is done numerically when the thermal constants are specified.) Calculation of temperatures by this method neglects two effects introduced by motion of material. Heat is transported as hotter mobile material moves toward the colder barrier. Also, displacements of the liquid redistribute the sources of latent heat. These effects are small when little motion is involved. The results of these effects will be discussed below.

The integration procedure takes the position of a parcel at the time of the step, computes a velocity from the present temperature and previous concentration information, and moves the parcel a distance equal to the velocity times the time increment in the step, if the comparison of positions reached by the present velocity and that used in the previous step is within the stability limits noted above. If the limits are exceeded, the increment is halved without moving the parcels until the discrepancy becomes less than $10^{-5} \times L$. Specific velocity formula and material constants used are discussed in the appropriate sections below.

\section{Formation of Eucritic Cumulates}

The eucrite parent body has produced a suite of achondritic meteorites which has been the object of intensive study. It has generally been agreed that the eucrites represent the disruption products of solidified lava flows or shallow intrusives [Mason. 1962; Duke and Silver, 1967]. Igneous textures may occasionally be seen through the mechanical overprint of brecciation, for example, in Ibitira, Juvinas, and Stannern. In a recent synthesis of information on the experimental petrology and origin of the eucrites, Stolper [1977] concluded that eucritic melts had the composition of a peritectic liquid in equilibrium with olivine, pyroxene, plagioclase \pm metal \pm spinel in the composition space containing eucrites. By inference, the eucrite compositions conspicuously oversaturated with olivine and pigeonite are not representative of liquids but are liquids with admixtures of pigeonite. Since pigeonite is denser than eucritic liquids, we investigated the concentration of pigeonite in terms of a model of cumulate formation. This problem has been treated in the study of small lunar and terrestrial magma bodies [Walker et al. 1976, 1977] and is adapted to the present study of a small eucrite magma body.

The relevant expression for velocity $(V)$ of crystals sinking to form cumulates is a modification of Stokes' law:

$$
V=\frac{2 g r^{2} \Delta \rho}{9 K \eta}(1-\phi)^{4.65}
$$

where $g$ is the acceleration of gravity, $r$ is the radius of the crystals, $\Delta \rho$ is the density contrast between crystal and melt, $K$ is a geometric factor which corrects for departure of crystal shapes from spherical, $\eta$ is the liquid viscosity, and $\phi$ is the fraction of crystalline material present in the melt which enters the exponential factor that modifies the effective viscosity for crystal sinking. A discussion of the relative merits of various possible formulations of (3) may be found in Walker et al. [1976].

The object of the exercise is to determine the minimum $g$ required to produce the concentration of pyroxene observed in the actual meteorites. For illustrative purposes we shall try to produce the accumulation of pyroxene found in Binda from a liquid similar in composition to the Juvinas eucrite. (Binda has about 50\% more pigeonite than Juvinas.) Pigeonites in Binda have diameters up to approximately $5 \mathrm{~mm}$, so we shall take $r$ as $2.5 \mathrm{~mm}$, and, since the crystals are reasonably symmetrical, $K$ will be taken as 1 . Although the pigeonites undoubtedly could have grown during the cooling event leading to their concentration, we shall take the size as a constant $r$ of $2.5 \mathrm{~mm}$ during the process. This treatment leads to a maximum amount of accumulation for fixed values of the other parameters and thus yields a minimum estimate of $g$. This treatment may be physically justified, since the eucrite liquids were saturated with pigeonite and such large crystals could have been present at the start of the accumulation process.

The viscosity and density of the liquid can be calculated by the methods of Bottinga and Weill $[1970,1972]$. The temper- 
ature dependence of liquid viscosity is strong and is retained in the numerical integrations, whereas the density contrast between crystals and liquids has a small temperature dependence which is neglected. The density of pigeonite is calculated from the cell dimensions of Robinson et al. [1977] and Smyth [1974] and the composition given by Ishii et al. [1976]. The resulting $\Delta \rho$ is taken as $0.5 \mathrm{~g} / \mathrm{cm}^{3}$ independent of temperature. The fraction of solidification of eucrite magmas as a function of temperature has been determined by Stolper [1977] and is approximated here as a linear increase from liquidus to solidus. The other parameters which need to be specified in order to perform the integrations are the thermal constants, the length $L$ characteristic of the dimensions of the magma body, and $g$. We have taken the thermal parameters of magma and country rock substrate to be the same (thermal diffusivity 0.01 $\mathrm{cm}^{2} / \mathrm{s}$, heat capacity $0.3 \mathrm{cal} / \mathrm{g} \cdot \mathrm{deg}$ ) except that the magma liberates $80 \mathrm{cal} / \mathrm{g}$ of latent heat of crystallization between $1175^{\circ}$ and $1075^{\circ} \mathrm{C}$.

With these parameters as input, we have calculated the concentration $(X)$ of pigeonite by accumulation that would be expected for range of values of the other free parameters: $g$ and magma body size. The results are shown in Figure 2 . We assume the country rock substrate density of $\sim 3.4 \mathrm{~g} / \mathrm{cc}$ is representative of the planet and the planet radius is scaled to $\mathrm{g}$ on this basis in Figure 2. The shaded upper left portion of the figure above the line labeled 'magma = planet' indicates physically impossible configurations where the magma body is bigger than the planet on which it resides. The rest of the diagram is contoured for the values of $X$ calculated in the numerical integrations. It is seen that either larger $g$ or larger magma body size leads to a greater amount of concentration. The former result arises because increasing $g$ stimulates crystal mobility leading to concentration. The latter result arises because increasing magma body size increases the cooling time, allowing more time for crystal accumulation to occur. The contours have a slope of -1 in Figure 2, indicating that

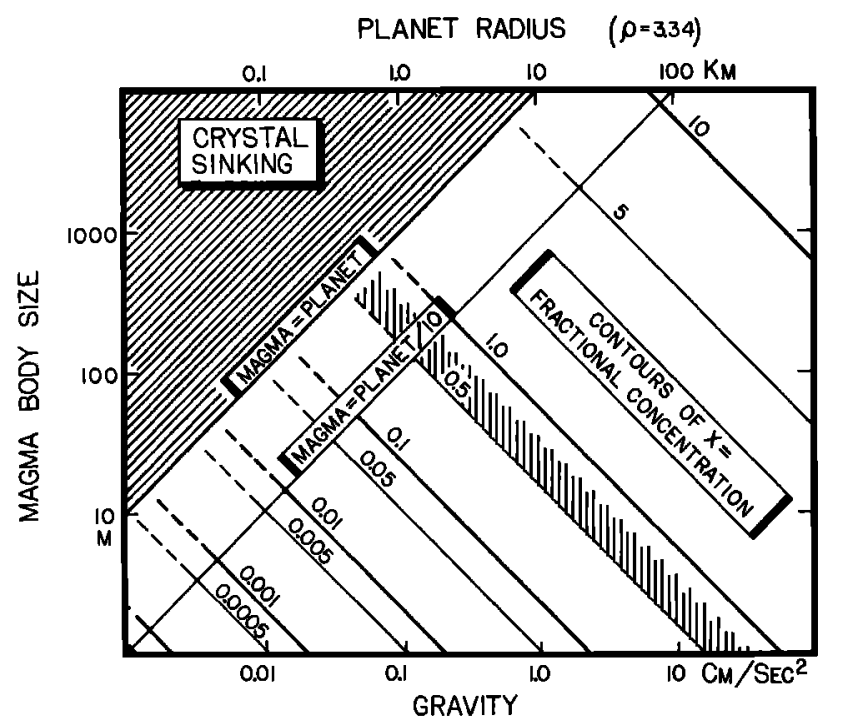

Fig. 2. Contours of fractional concentration of pigeonite produced by accumulation during crystal sinking in a eucritic magma as a function of $g$ and $L$ calculated by (2). Planet radius is scaled to $g$ for an assumed mean planet density of $3.34 \mathrm{~g} / \mathrm{cm}^{3}$. Diagonal lines from upper right to lower left are the loci of points with fixed magma/planet size ratio. Vertical shading near contour $X=0.5$ corresponds to the accumulative concentration of pigeonite observed in Binda relative to Juvinas (about 1.5 times). increasing either $g$ or magma body size by the same factor from some point on the $g$ versus magma body size plot leads to the same amount of accumulation. This result may be approximately understood in terms of the dependence of the cooling time on the square of magma body size. For an order of magnitude increase of magma body size, the distance crystals must move to achieve a particular concentration increases an order of magnitude, while the cooling time available for mobility increases by 2 orders of magnitude. On balance, then, for the same velocity parameters, the gain in mobility leading to extra concentration (rather than just covering the extra distance) is an order of magnitude. This is equivalent to the direct effect on mobility of an order of magnitude increase in $g$.

We can see in Figure 2 that the minimum $g$ for any $X$ corresponds to the largest magma body permissible. So to proceed, we shall consider the values of $X$ reached as a function of $\boldsymbol{g}$ for magma bodies which compose a substantial part of the planet. Lines of fixed ratio of planet to magma body size are diagonal with slope 1 on Figure 2 . We shall arbitrarily consider such a line with magma body thickness to planet radius of $\$$. This ratio of magma to planet size represents an approximate upper limit to magma body size which can be produced by wholesale extraction of a $10 \%$ partial melt from a planetary interior. We could consider larger magma bodies with respect to planet size by discarding the constraint of how much melt is reasonably available, but this would be impractical. For magma bodies of the same order as planet size, the discrepancy between the half-space geometry presumed in the calculations and the spherical geometry of reality becomes troublesome. Furthermore, the decrease of $g$ with depth becomes significant enough to offset the gain in integrated motion produced by larger size, so no useful purpose would be served by making the magma size the same order as the planet size.

The values of $X$ as a function of $\boldsymbol{g}$ from Figure 2 for magma/ planet $=\frac{1}{30}$ are shown as the heavy curve on Figure 3 . The lower portion of this curve in the range of very small concentrations is linear with a slope of 2 (note scales on different axes). In the limit of small concentrations, increasing planet radius (i.e., $g$ ) by a factor of 10 leads to a factor of 100 increase of $X$ because we are scaling the magma body size to the planet size. One factor of 10 of increase comes directly from $g$, the other from the increase of magma body size. This simple relationship breaks down at higher concentrations where the

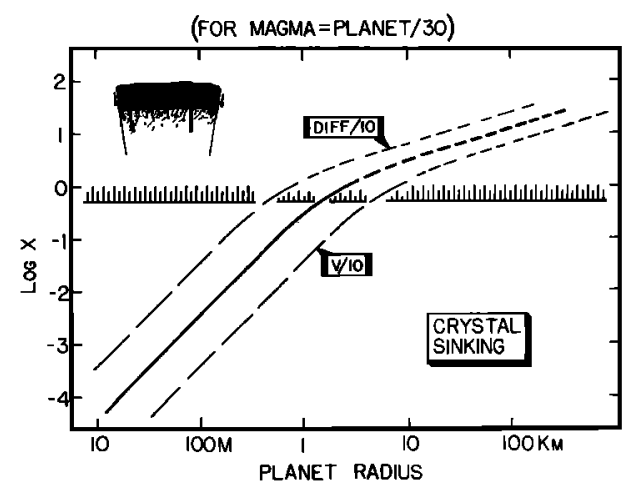

Fig. 3. Concentration of pigeonite achieved versus planet radius for fixed magma/planet ratio of so. Curves for order of magnitude changes in velocity and thermal diffusivity are shown. Horizontal hatched line is concentration of pigeonite observed in Binda relative to Juvinas. Planets about $1 \mathrm{~km}$ in radius will produce the observed concentration. 
curve is seen to fall significantly below a projection of the curve from small $X$ to large $X$. This negative deviation is a reflection of the negative feedback intrinsic to the crystal settling process. The greater the concentration becomes, the larger the fraction of crystals $(\phi)$ becomes, and so the velocity expression in (3) is reduced exponentially as accumulation proceeds.

For large motions and concentrations, the transport of heat and redistribution of latent heat sources will influence the temperature calculation and hence the cooling time available for mobility. Sinking of hot crystals warms the surroundings and thereby slows the local temperature drop. (Paradoxically, this mode of heat transport can lead to a higher thermal gradient and heat flux and hence shorter total cooling time for the whole magma.) However, crystal sinking displaces liquid with its latent heat, thereby contributing to a more rapid local solidification. This is to say that heat transport and displacement of latent heat sources have a compensatory effect on the local rate of solidification. Although we have ignored both these effects in our calculations of temperature, we can deduce the direction in which they perturb the results shown in Figure 3. If the heat transport effect is dominant and cooling is retarded, the negative deviations seen at high concentrations will be reduced as extra mobility and opportunity for concentration is experienced. If latent heat source displacement is predominant, the negative deviations at high concentrations will be enhanced. Approximate calculations appropriate to the solution shown in Figure 2 suggest that the displacement of latent heat sources is slightly more powerful than the compensating heat transport effect. Whichever effect predominates, the conclusions of the next paragraph are not materially changed, since the conclusions depend on relatively small factors of concentration.

The hatched horizontal line in Figure 3 indicates the amount of pigeonite concentration seen in Binda relative to liquid composition Juvinas. The intersection of this line with a $g-X$ curve indicates that the eucrite parent planet need only be on the order of $1 \mathrm{~km}$ in radius to produce the concentration of pyroxene seen in Binda, if a substantial fraction of the planet was involved in the magma body. Also plotted on Figure 3 are curves which result from adjustment of the input parameters. It can be seen that if the velocity expression were in error by an order of magnitude, the result would only be different by $10^{1 / 2}$. The same is true for an order of magnitude error in the thermal diffusivity. Consequently, if the treatment has any relevance to the natural process, the results are sufficiently insensitive to small errors in the input parameters so that order of magnitude estimates of a minimum $g$ can probably be made with some confidence, i.e., the planet must be larger than a bread box, but the dimensions of large asteroids are not required to induce cumulate formation.

The model of cumulate formation by gravitationally induced crystal sinking used here is quite obviously inadequate to describe the evolution of lunar and terrestrial magma bodies of the size postulated here for the eucrite planet because of the large amount of convective mass transfer. We have not explicitly considered this problem in the present formulation because we postulate that we are dealing with the lower portion of a magma which is cooled from below and hence is convectively stable. This treatment seems to be adequate in dealing with small lunar and terrestrial magma bodies, where large convective flow is minimal. Furthermore, calculation of the Rayleigh number for the sort of magma body envisioned on the eucrite planet suggests that convective instability will not be encountered until the planet is in the kilometer range in radius. That is to say, our cumulate formation model probably is adequate for the size ranges indicated in the results. Had larger planets and magma bodies been indicated, then fluid convection would have substantially enhanced simple crystal sinking. Our calculations place only minimal constraints on the size of the eucrite parent planet. It need only approach 1 $\mathrm{km}$ in radius before simple crystal sinking or convection will produce cumulates. This is only a minimum constraint, since any larger size will also be sufficient. Clearly, the existence of eucrite cumulates does not exclude many directly observed solar system objects as candidates for the eucrite parent planet.

Another point of discussion concerns the plausibility of our initial condition-a small planet having its outer portion molten early in its history. Although this may seem implausible at face value, something of the sort seems to have occurred early in lunar history. Furthermore, our understanding of the heat sources available for melting small objects very early in solar system history is so inadequate that the proposed conditions can scarcely be considered less plausible than others we might suggest. We should keep in mind that the object of the present exercise is to discover a minimum $g$ required for production of cumulates, and we have so chosen our magma/planet size ratio to insure the $g$ we discover is a minimum. These may not be the conditions which actually obtained-we may really be dealing with small pools of magma internally generated on a larger object-but this set of conditions cannot be dismissed and must be retained in a discussion designed to determine a minimum $g$.

\section{Segregation of Eucrite Melts}

The existence of eucrite melts with differentiated, distinctly noncosmic compositions implies the operation of chemical fractionation even before the events of the last section transpired. Stolper [1977] has suggested that this fractionation is the result of producing eucrites by direct partial melting of a primitive source region containing olivine, low-calcium pyroxene(s), and plagioclase \pm metal \pm spinel. Indeed, a number of recent papers have treated such a model of direct partial melting in terms of the minor elements and have succeeded in rationalizing eucrites as the melting products of a roughly chondritic source region [Consolmagno and Drake, 1977; Morgan 'et al. 1978; $M a$ and Schmitt, 1977]. The conclusion reached by these authors is that the eucrites could represent the liquid produced by a partial melting event of about $5-10 \%$ of a nearly chondritic source region. The problem that we explore, then, is what are the necessary conditions which must exist in order to extract successfully a small amount of melt dispersed throughout a source region? In terms of our previous approach, this is equivalent to asking what range of parameters will lead to sufficient integrated melt mobility and hence concentration before cooling blocks the escape of melt.

The model for melt mobility used was originally proposed by Frank [1968] and has been expanded upon by Sleep [1974] and Turcotte and Ahern [1978]. Partial melting in the source region produces melt-filled porosity which interconnects to establish permeability. Buoyant forces cause melt to percolate along the interconnected channels. Lending plausibility to this model, Waff and Bulau [1977] have shown experimentally that small amounts of basaltic melt dispersed in a peridotitic matrix collect along grain boundaries and that although complete crystal face wetting does not occur at small degrees of melting, the dihedral grain-melt-grain angles are observed to be such 
that grain boundary channels may be reasonably inferred to interconnect and establish permeability.

The expression used for melt velocity is a modification of Darcy's law where the force driving the flow of melt in the porous, permeable source region in a gravity field derives from the density difference between crystals and melt:

$$
V=\frac{g \Delta \rho R_{c}^{2} f^{2}}{73.5 \eta}=\frac{k}{\eta}(\nabla \text { pressure })
$$

The parameters $g, \Delta \rho$, and $\eta$ are the same as in (3). The parameter $f$ is the fraction of melt present in the source region, and $R_{c}$ is the effective radius of crystals in the source region. The factor 73.5 relates porosity and grain size to the permeability $k$ for the geometry of the source region. We have used the crystalline geometry of truncated octahedra suggested by Frank [1968], but we assume the melt to be distributed on channels along 3-grain contacts which have equilateral triangular rather than circular cross sections. We have also assumed a Kozeny flow law. [Scheidegger. 1957] rather than the Poiseuille law assumed by Frank. These modifications give a numerical constant 73.5 which leads to higher velocities and hence lower $\boldsymbol{g}$ than the $\sim \mathbf{6 4 0}$ suggested by Frank. However, the dependence of velocity on $R_{c}{ }^{2}$ and $f^{2}$ remains the same.

We have used the treatment of Bottinga and Weill [1970, 1972] to calculate viscosity and density of the eucritic liquid. The temperature dependence of viscosity is retained in the integrations, whereas the temperature dependence of $\Delta \rho$ is not. The fraction of melting $(f)$ is initially taken as $5 \%$ (to correspond to eucrite Stannern) but is adjusted during the integrations both for previous integrated mobility leading to extra concentration and for temperature. The values of $L$, the characteristic thickness of the source region, and $g$ are unknown, and the calculations are done for a range of these values. The value of $\boldsymbol{R}_{c}$ is also unknown and must be explored, although intuition can be used as a guide. To find the minimum $g$, we should use the value of $\boldsymbol{R}_{c}$ most conducive to melt mobility, i.e., the largest one reasonable. Silicate crystals of size as large as $5 \mathrm{~cm}$ are rarely present in meteorites (cf. Estherville), although such large megacrysts are occasionally brought up from depth in terrestrial alkali basalts. The grain size of terrestrial lherzolites, thought to be material from the source region of terrestrial basalts, rarely approaches this size and is more commonly an order of magnitude smaller. We perform the calculations for values of $R_{c}$ of 2.5 and $0.25 \mathrm{~cm}$, which we infer to be the largest reasonable values. It will be seen that the answers derived can be adjusted very simply if any information on the actual value of $R_{c}$ should become available.

The thermal regime in the present problem is somewhat more complicated to specify than in the crystal settling problem. In the first place, since we do not know the details of the mineralogy and hence the melting interval of the source region, we can only approximately specify the melting interval and the amount of latent heat involved in the production of eucrite liquid. The proposed bulk compositions [Consolmagno and Drake, 1977; Morgan et al., 1978] for the source region, which contain a large excess of olivine in reaction relation with the liquid, suggest that eucrite liquids are produced near the solidus of the source region. The fact that these liquids represent $5-10 \%$ melting suggests that only a small fraction of the heat of fusion of the source region is involved. We have performed the calculations on the basis that $5 \mathrm{cal} / \mathrm{g}$ of latent heat of fusion of the source region are required to produce eucrite liquids over the temperature interval from $1165^{\circ}$ to $1175^{\circ} \mathrm{C}$. The results are fairly insensitive to this small heat of fusion and temperature interval; however, the size of temperature interval leading to $5 \%$ fusion is important in the following connection.

The emplacement of a magma body such as a flow or pluton can occur in rocks with which the magma has strong temperature contrast, but it is unlikely that a source region would be heated so that there was a large initial, discontinuous temperature contrast with the cooler overburden. In our calculations we have therefore reduced the temperature contrast so that the source region temperature approaches the overburden temperature. However, if the temperature contrast becomes of the order of the temperature interval involved in the $5 \%$ fusion, heat transfer between the two half-spaces will not result in solidification. Instead, the overburden becomes partially molten and the percolation process continues without a natural termination. Consequently, we are obliged to keep the initial temperature contrast a few times larger than the temperature interval in order to provide for a physically bounded problem. Undoubtedly, in a real situation, the partially molten source region grades continuously into a cooler, solid overburden. Although our initial conditions provide for a step function temperature discontinuity between the source region and the overburden, the melt parcels we consider start far enough away from this interface that it has degenerated to the more realistic continuous condition by the time our melt parcels approach it. The solution is explored for various values of the initial temperature contrast and is found to be essentially independent of temperature contrast for contrasts greater than $100^{\circ} \mathrm{C}$ (10 times the fusion interval).

Calculations of melt concentration have been performed as a function of $g$ and $L$, with the other parameters fixed. The result discovered in Figure 2 is found again: contours of $X$ have a slope of -1 on a $g$ versus $L$ plot. This is to be expected, since (3) and (4) have the same dependence on $g$ and the cooling problem has the same dependence on $L$ in both cases. If we then choose a fixed ratio of planet to source region size, we can directly plot $X$ versus $g$ (or planet size). Since we should put the source region beneath at least an equal thickness of cooler overburden to be consistent with our simplified halfspace model, we have arbitrarily set the ratio of planet to source region size at 10 .

The resulting plot of $X$ versus planet size is shown in Figure 4. The curve for very low concentrations of melt is linear with a slope of 2 for the same reasons as in Figure 3. Physically, this low concentration corresponds to situations of low melt mobility where melt solidifies and is trapped near its site of generation in the source region. In contrast to Figure 3, the deviation of the curve from an extrapolation of the low concentration regime is strongly positive rather than negative. This positive deviation is a reflection of the positive feedback between melt concentration and melt mobility which enters (4) as $f^{2}$. Whereas the crystal settling process has an intrinsic negative feedback, the melt accumulation process has an intrinsic positive feedback even when both processes occur in a regime of falling temperature.

The hatched horizontal line in Figure 4 corresponds to a concentration factor of $\mathbf{2 0}$ or complete separation of the original $5 \%$ melt from residual crystalline source region. Extension of the curves of $X$ above this line is physically unrealistic. Actually, the use of (4) to calculate $X$ approaching this line (above a $\log X \approx 1$ ) is also somewhat unrealistic, since at such high fractions of melt $(\sim 30-50 \%)$, melt floods the network of 


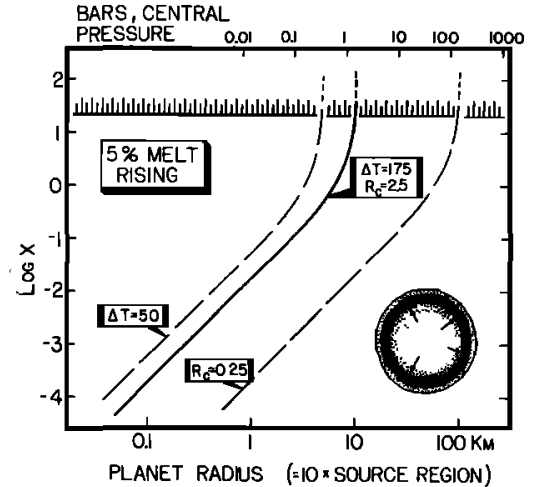

Fig. 4. Concentration of eucrite liquid achieved versus planet radius for fixed source region/planet ratio of $\frac{1}{\mathrm{t}}$. Initial melt concentration is 5\%; hatched horizontal line is concentration for complete separation of melt $(20 X) . \Delta T$ is initial temperature contrast between half-spaces in degrees Celsius, and $R_{\mathrm{c}}$ is crystal radius in source region in centimeters. Planet should be in 10-100 km range to segregate eucrite liquid. Maximum hydrostatic pressure in planet, at its center, is scaled to planet radius for assumed mean density of $3.34 \mathrm{~g} / \mathrm{cm}^{3}$.

channels so that a porous, solid medium becomes a liquid medium with suspended crystals. Although (3) would be more appropriate to use for the separation velocity of crystals and melt in this situation, (3) will give a higher velocity than (4), leading to a more steeply sloping upper portion of the curve. We have also ignored incorporation of heat transport and latent heat source displacement effects caused by moving liquid. In contrast to crystal settling, where the effects compensate one another, both effects lead to a decrease in the local solidification rate of percolating liquid. As a result, extra segregation can occur, and so the upper portion of the curve of Figure 4 should be steepened by these effects as well. Since the curve already approaches vertical, there is little point in pursuing any of these refinements in this discussion.

The steepness of the upper portion of the curve in Figure 4 means that small increases in $g$, or in any other parameter leading to increased melt percolation velocity, lead to very large increases in the concentrated accumulation $(X)$ of melt in this range. It is seen that melt accumulation is a runaway process if the fraction of melt begins to increase appreciably (about $10 \%$ in this example) at some stage of the accumulation process. This instability is the result of the positive feedback between percolation velocity and the extent of melt accumulation which was noted above. It appears from Figure 4 that this instability will be encountered with the resulting successful segregation of eucrite liquid if the planet radius is $\sim 10 \mathrm{~km}$ for an $R_{c}$ of $2.5 \mathrm{~cm}$. This grain size for the source region may be unrealistically large, so the curve for the slightly more plausible $R_{\mathrm{c}}$ of $0.25 \mathrm{~cm}$ is also shown. Decreasing the grain size an order of magnitude increases the planet size required for segregation an order of magnitude to $\sim 100 \mathrm{~km}$. This direct relation is general and can be used to modify the conclusions if $R_{c}$ should become known by other means, since curves produced by variation in $R_{c}$ remain congruent when shifted laterally. On the other hand curves produced by variation of the temperature contrast $(\Delta T)$ between source region and overburden are not exactly congruent, although they are nearly so. The curves for $\Delta T>100^{\circ} \mathrm{C}$ are all very close to one another, and $\Delta T=$ $175^{\circ} \mathrm{C}$ is arbitrarily shown. For comparison, a curve with $\Delta T$ as low as $50^{\circ} \mathrm{C}$ is also shown. A smaller planet size is required because lowering the temperature contrast makes extra cooling time available for melt motion.
The calculations suggest that the eucrite planet radius should have been at least in the $10-100 \mathrm{~km}$ range in order to be able to segregate small percentages of melt from a source region before it was solidified in place. There is obviously more uncertainty about this conclusion than about the conclusion based on the crystal settling analysis because we are not able to specify the grain size or the thermal structure of the source region with much confidence. But it is probably safe to conclude that the accomplishment of melt segregation implies a larger minimum $g$ and planet size for the eucrite parent planet than the accomplishment of cumulate formation.

Some discussion is necessary of the model for melt accumulation we have used: percolation in a porous medium in the absence of tectonic perturbations. It would be a serious omission to exclude tectonic perturbations on a planet such as the earth where solid state convection is apparently churning the source regions of basalts. Indeed, Turcotte and Ahern's [1978] interest in the porous flow model centers on the migration of liquid produced as the upwelling limb of a convection cell is driven across a melting curve. In contrast, we have investigated the accumulation of liquid produced in the absence of such melting mechanisms, since solid state convection is unlikely to occur on bodies of the size we are considering (e.g., a $10,000^{\circ} \mathrm{C}$ temperature drop would be needed to drive convection on a $100-\mathrm{km}$ planet). Other sorts of tectonic perturbations which might promote melt segregation, such as localized shearing, are ultimately dependent on the gravity field for their origin. The differential stress available to operate such mechanisms cannot be very much more than a few times the hydrostatic pressure generated by the gravity field. Figure 4 shows the maximum hydrostatic pressure of a planet as a function of radius: only 170 bars for a $100-\mathrm{km}$-radius object. This suggests that tectonic mechanisms of melt accumulation are minimal on such small objects and that we must rely directly on local buoyant forces to extract melt.

A hidden assumption in the model of melt migration is that withdrawal of melt is compensated by the plastic response of the porous medium (i.e., we must avoid trying to squeeze fluid from a rigid sponge). The major silicate constituent of our porous source region is apparently olivine [Stolper, 1977; Consolmagno and Drake, 1977; Morgan et al., 1978], and we will treat the rheology of our source region as that of olivine to a first approximation. Ashby and Verrall [1978] have considered the various competing micromechanisms of flow in olivine and have produced maps of deformation behavior as a function of temperature, pressure, and shear stress. We can use these maps to infer the time scales on which the source region behavior is fluid enough to allow melt extraction. For planets of the order of $100 \mathrm{~km}$ in radius, stresses of a few tens of bars are produced, and, at the source region temperatures required, the deformation rates are expected to be of the order of $10^{-\theta}-10^{-12} / \mathrm{s}$. Since the source region in a planet this size has a cooling time for melt mobility of the order of $10^{7}$ years $\left(\sim 10^{14} \mathrm{~s}\right.$, determined from the integrations of (4)), the source region is squishy enough by more than 4 orders of magnitude to allow melt extraction. Hence, for $100-\mathrm{km}$ planets, the hidden assumption is prabably valid. However, in $10-\mathrm{km}$ objects which generate stresses of only a few tenths of a bar in a source region which has a cooling time for melt mobility of $\sim 10^{5}$ years $\left(\sim 10^{12} \mathrm{~s}\right)$, the deformation rates are expected to be of the order $10^{-13}$ $10^{-14} / \mathrm{s}$. Clearly for planets of this order of size, the hidden assumption is only marginally, if at all, valid, and melt extraction by buoyant forces will be inhibited by the effective rigidity of the source region. A further complication in these smaller 
objects is that void spaces in the source region cannot be fully closed on the time scale available. Retention of void spaces may further complicate melt migration by introducing capillarity barriers to the melt and upsetting the density contrasts which drive the percolations. For these reasons, then, our treatment of melt accumulation only gains validity for planets with radius as large as the $10-100 \mathrm{~km}$ range. It is of interest to note that the size required to give the source region sufficient plasticity to allow the melt to escape is of the same order as the size required to make the melt mobile enough to escape. Although the former condition is required for the validity of the analysis leading to the second, the conditions are independent, and the minimum size arrived at by each argument could be different. In any case, we can probably take the $10-100 \mathrm{~km}$ radius as a firm lower limit on planet size, because even though this limit from the melt mobility analysis has large uncertainties generated by the unconstrained parameters, we find that a similar lower limit is required to make the analysis valid in the first place.

These insights into the size of the eucrite parent body suggest that if it is more or less intact today as suggested by Stolper [1977] and Consolmagno and Drake [1977], it must be one of a rather limited number of bodies in the solar system. This analysis would seem to exclude the large collection of smaller asteroids. The results here are consistent with and strengthen the arguments made by $\mathbf{M c C o r d}$ et al. [1970], Stolper [1977], and Consolmagno and Drake [1977] that identify the eucrite parent body as asteroid 4 Vesta, although there may be dynamical problems associated with deriving eucrites from Vesta [Wetherill, 1974]. Whatever the merits of the arguments for or against Vesta being the actual parent body of the eucrite meteorites, the important point to emerge from this discussion is that the parent body of the eucrites has to be of this order of size and cannot be a very small object.

\section{Stability of Melt in the Terrestrial LOW-VELOCITY ZONE}

The layer of low seismic velocity and high attenuation beneath the earth's lithosphere is believed to be a zone in which modest amounts of melt are present [Anderson and Sammis, 1970; Solomon, 1972]. If this is the case and if the melt is present on a permeable, interconnecting network, then the treatment of the last section can be applied to learn under what conditions the melt might be expected to segregate.

Let us assume that the melt forms an interconnecting net [Waff and Bulau, 1977; Shankland and Waff, 1977] and ask whether the instability noted in the last section leading to runaway segregation will be encountered. The values of $g$ and $L$ are not free parameters as they were in the previous section, and the value of $R_{c}$ is unlikely to be less than $1 \mathrm{~mm}$ if the grain size of lherzolite nodules from the earth's mantle is any guide to the minimum' grain size in the source regions of the alkali basalts and kimberlites which bring them to the earth's surface. The viscosity of melt in the low-velocity zone (LVZ) is less well constrained than it is in the previous section. Solomon's [1972] analysis suggested that it may be as high as $10^{8} \mathrm{P}$, but this is difficult to reconcile with petrologic observation. The basalts which erupt typically have viscosity $\sim 10^{2}-10^{3}$ at $\sim 1200^{\circ} \mathrm{C}$. Furthermore, the composition of the low melting fraction at the pressures of the low-velocity zone should be considerably more olivine-normative and less viscous than the common basalts erupted at the surface [O'Hara, 1968]. In addition, Kushiro et al. [1976] have suggested that increasing pressure lowers the viscosity of basalt even without considering the change in composition which must occur. Furthermore, the likely inclusion of water in the low melting fraction would tend to lower the viscosity. Consequently, we consider $10^{2} \mathrm{P}$ to be a more probable upper limit to melt viscosity than $10^{\circ} \mathrm{P}$. The value of the density contrast between melt and crystals is probably near $0.7 \mathrm{~g} / \mathrm{cm}^{\mathrm{g}}$.

Using these parameters, we can calculate the behavior of the LVZ by the methods of the previous section to see if the instability leading to runaway melt segregation is encountered. We have performed these calculations and find that the instability is encountered for melt fractions greater than $\sim 0.1 \%$. (The fact that the instability is encountered could have been anticipated by reference to Figure 4 , where $g$ and $L$ appropriate to the $\mathrm{LVZ}$ have exceeded the instability limit by several orders of magnitude, albeit for a slightly different material.) This conclusion is conservative when one considers that the calculations employ a thermal regime of conductive cooling with no external heat sources, whereas the LVZ undoubtedly is influenced by heat flow from the earth's interior and hence cools more slowly than predicted by the calculations. Therefore, extra time of differentiation is available to lead to the instability, and the stabilization of melt fractions as low as $0.1 \%$ may be artificial-they are probably unstable also.

Instead of using a model of conductive cooling of halfspaces for the LVZ, it might be an improvement to consider the LVZ to be in roughly a thermal steady state with heat loss to the lithosphere balanced by heat flow from below. The question then becomes one of whether melt will segregate before the steady state temperature declines with the time constant of the earth to the point where the LVZ could no longer exist as a zone of partial melting. In a steady state temperature regime, we no longer consider local heat loss to be the limiting process in retarding segregation, since any fraction of melt should percolate out given long enough. As we have seen that the $\mathrm{LVZ}$ may be expected to be grossly unstable with respect to draining itself of melt, the issue is not whether segregation will occur but how long does it take.

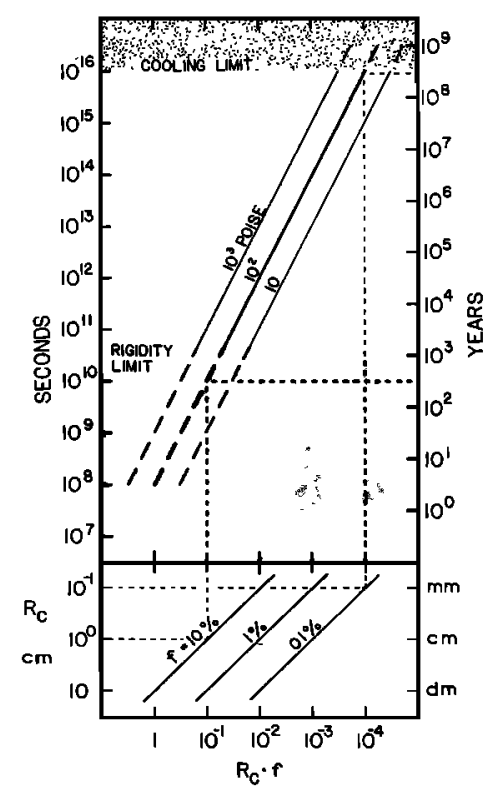

Fig. 5. Time for liquid moving with velocity given by (4) to traverse the $L V Z$ versus $\boldsymbol{R}_{c} \times f$. Lower portion of figure gives $\boldsymbol{R}_{c} \times f$ from $\boldsymbol{R}_{c}$ chosen and appropriate $f$ contour. Solutions for various liquid viscosities are contoured on the upper part of the figure. For short times, rigidity of the LVZ limits the discharge rate; for long times, cooling of the earth limits the discharge rate. 
We can produce order of magnitude answers to this question fairly simply without recourse to the numerical integration procedures of the previous section by calculating the time needed for a parcel of liquid to traverse the LVZ if the velocity is given by (4). This time is shorter than the time required to completely drain the $\mathrm{LVZ}$. Residual melt in declining amounts moves with ever decreasing velocity as $f$ drops, so the last stages of the draining process are quite slow. The time we are calculating is approximately that necessary to produce a substantial accumulation of segregated liquid beneath the lithosphere. For this calculation, the $\mathrm{LVZ}$ is taken as $100 \mathrm{~km}$ thick. The temperature is assumed to be steady state and to exceed the solidus temperature of the mantle in the LVZ. The actual temperature profile is not known, nor is the exact position of the solidus relative to this profile. Consequently, the viscosity and melt fraction parameters are taken as constant in each calculation but are varied from calculation to calculation.

Figure 5 shows the results of these calculations of the length of time required for melt to traverse the $L V Z$ as a function of the other parameters. It can be seen that a LVZ with $10 \%$ melt is a strictly ephemeral feature and would very quickly reduce itself to a segregated condition for any reasonable value of crystal size and melt viscosity. For instance, $10 \%$ melt dispersed throughout an LVZ of centimeter sized crystals will drain in a few centuries for a viscosity of $100 \mathrm{P}$. The curves in Figure 5 are subject to two additional considerations. At very high calculated drainage rates, the effective rigidity of the solid portions of the LVZ begins to become an additional retardant to porous flow of liquid, as discussed above. Using the results of Ashby and Verrall [1978] for the earth's mantle, deformation rates of $10^{-10}-10^{-12} / \mathrm{s}$ are expected for the depth and differential stress driving the percolation. Therefore the LVZ rigidity can act as a brake to segregation for drainage times up to a few millenia. The rigidity does not stop segregation; it merely slows it so the lines in Figure 5 should have a shallower slope at the bottom end. A second consideration affects the upper ends of the lines. For time scales of $10^{8}-10^{9}$ years, cooling of the earth may lower the steady state temperature such that segregation will not occur and the curves would end.

Reference to Figure 5 suggests that if the LVZ is to persist on its own on a geologic time scale, melt fractions must be in the less than 1-0.1\% range. Anderson and Spetzler [1970] addressed the question of how much melting was required to explain the seismic properties of the LVZ by using the Eschelby-Walsh theory of attenuation. They concluded that melt fractions of this order were sufficient. Recently, $O^{\prime}$ Connell and Budiansky [1977] analyzed viscoelastic dissipation mechanisms in fluid-saturated cracked rocks and also concluded that such small fractions of melt might be capable of explaining the seismic properties of the LVZ. However, both studies considered geometries of liquid distribution which approximate surface films on grain boundaries. The conclusion that very small amounts of melting are sufficient to explain LVZ seismic properties is sensitive to the geometric model used for melt distribution. Even within the model of melt as films or in cracks, the conclusion depends on the aspect ratio of the films or cracks. The work of Waff and Bulau [1977] suggests that melt distribution should not be as films but as tubules on three-grain contacts. The effect of tubule geometry of melt on seismic attenuation has not been quantitatively explored as yet, but larger fractions of melt will probably be required to explain LVZ attenuation than is the case for melt films. Consequently, it is not clear at this time whether melt fractions in the $0.1-1 \%$ range can explain the attenuation properties of the $L V Z$ if the tubule geometry of Waff and Bulau [1977] is correct.

Arndt $[1977 a, b]$ has presented experimental evidence that melt distribution may be as films, although the central issue in those reports was not whether this was the equilibrium textural distribution, as advocated in the Waff-Bulau model of tubules which we have adopted. Arndt made the important point that the exponent relating melt fraction to permeability (and to melt velocity) increases as one goes to a film geometry. Therefore a larger fraction of melt could be accommodated in the LVZ as films rather than tubules because melt escapes less easily with a decreased velocity. Furthermore, the velocities we have calculated on the basis of the Waff-Bulau tubule model may be too high, since there is no assurance that the tubules maintain a constant cross section along their length $(H$. S. Waff, personal communication, 1978), even if they do have the general form and interconnectedness we have supposed. This effect, which could lower melt velocities in the tubule geometry we used, also would allow a greater fraction of melt to remain in the $L V Z$.

Both calculations involving the fraction of melt in the LVZ (the amount that can be in mechanically stable residence and the amount that is required to explain the seismic properties) are dependent upon the uncertainties in the actual geometry of melt distribution. Although it appeared above that slightly more melt may be required to explain the seismic properties than would be stable on a geologic time scale, this incongruity cannot be considered significant when measured against our uncertainty about the melt geometry. One possible interpretation is that there is no discrepancy. If this is the case, then the seismically inferred melt fraction in the LVZ could be understood as a drainage residue. The implication is that if larger amounts of melt are produced or introduced into the $\mathrm{LVZ}$, they are drained down to the stable residual levels seen fairly quickly.

Acknowledgments. We thank H. S. Waff, R. A. Verrall, and J. L. Ahern for supplying preprints of their work in advance of publication and H. S. Waff, N. H. Sleep, M. J. Drake, B. Hager, R. J. O'Connell, and an anonymous reviewer for comments on the manuscript. This work was supported by NASA grant NGL-22-007-247 and by the Committee on Experimental Geology and Geophysics of Harvard University and is contribution 28 of the Basaltic Volcanism Study Project organized and administered by the Lunar and Planetary Institute/Universities Space Research Association under NASA contract NSR 09-051-001.

\section{REFERENCES}

Anderson, D. L., and C. Sammis, Partial melting in the upper mantle, Phys. Earth Planet. Interiors, 3, 41-50, 1970.

Anderson, D. L., and H. Spetzler, Partial melting and the low velocity zone, Phys. Earth Planet. Interiors, 4, 62-64, 1970.

Arndt, N. T., Ultrabasic magmas and high-degree melting of the mantle, Contrib. Mineral. Petrol., 64, 205-221, $1977 a$.

Arndt, N. T., The separation of magmas from partially molten peridotite, Carnegie Inst. Wash. Yearb., 76, 424-428, $1977 b$.

Ashby, M. F., and R. A. Verrall, Micromechanisms of flow and fracture, and their relevance to the rheology of the upper mantle, Phil. Trans. Roy. Soc. London, Ser. A, 288, 59-95, 1978.

Bottinga, Y., and D. F. Weill, Densities of silicate liquid systems calculated from partial molar volumes of oxide components, $A$ mer. J. Sci., 269. 169-182, 1970.

Bottinga, Y., and D. F. Weill, The viscosity of magmatic silicate liquids: A model for calculation, Amer. J. Sci., 272, 438-475, 1972.

Consolmagno, G. J., and M. J. Drake, Composition and evolution of the eucrite parent body: Evidence from rare earth elements, Geochim. Cosmochim. Acta, 4I, 127I-1282, 1977. 
Duke, M. B., and L. T. Silver, Petrology of eucrites, howardites and mesosiderites, Geochim. Cosmochim. Acta, 31, 1637-1665, 1967.

Frank, F. C., Two-component flow model for convection in the Earth's upper mantle, Nature, 220, 350-352, 1968.

Ishii, T., M. Miyamoto, and H. Takeda, Pyroxene geothermometry and crystallization: Subsolidus equilibration temperatures of lunar and achondritic pyroxenes, in Lunar Science VII, pp. 408-4I0, Lunar Science Institute, Houston, Tex., 1976.

Jaeger, J. C., The temperature in the neighborhood of a cooling intrusive sheet, Amer. J. Sci., 255, 306-318, 1957.

Kushiro, I., H. S. Yoder, and B. O. Mysen, Viscosities of basalt and andesite melts at high pressures, J. Geophys. Res., 81, 6351-6356, 1976.

Ma, M.-S., and R. A. Schmitt, Possible source materials for eucritic achondrites based on multi-linear regression analysis of trace element data, Meteoritics, $11,324-325,1976$.

Mason, B., Meteorites, John Wiley, New York, 1962.

McCord, T. B., J. B. Adams, and T. V. Johnson, Asteroid Vesta: Spectral reflectivity and compositional implications, Science, 168 . 1445-1447, 1970.

Morgan, J. W., H. Higuchi, H. Takahashi, and J. Hertogen, A "chondritic' eucrite parent body: Inference from trace elements, Geochim. Cosmochim. Acta, 42, 27-38, 1978.

O'Connell, R. J., and B. Budiansky, Viscoelastic properties of fluidsaturated cracked solids, J. Geophys. Res., 82, 5719-5735, 1977.

O'Hara, M. J., The bearing of phase equilibria studies in synthetic and natural systems on the origin and evolution of basic and ultrabasic rocks, Earth. Sci. Rev., 4, 69-133, 1968.

Robinson, P., M. Ross, G. L. Nord, J. R. Smyth, and H. W. Jaffe, Exsolution lamellae in augite and pigeonite: Fossil indicators of lattice parameters at high temperature and pressure, Amer. Mineral. . 62, 857-873, 1977.
Scheidegger, A. E., The Physics of Flow Through Porous Media, MacMillan, New York, 1957.

Shankland, T. J., and H. S. Waff, Partial melting and electrical conductivity anomalies in the upper mantle, J. Geophys. Res., 82, 5409$5417,1977$.

Shaw, H. R., Comments on viscosity, crystal settling, and convection in grąnitic magmas, Amer. J. Sci., 263, 129-152, 1965.

Smyth, J. R., The high temperature crystal chemistry of clinohypersthene, Amer. Mineral., 59, 1069-1082, 1974.

Sleep, N. H., Segregation of magma from a mostly crystalline mush, Geol. Soc. Amer. Bull., 85, 1225-1232, 1974.

Solomon, S. C., Seismic wave attenuation and partial meiting in the upper mantle of North America, J. Geophys. Res., 77, 1483-1502, 1972.

Stolper, E., Experimental petrology of eucritic meteorites, Geochim. Cosmochim. Acta. 41, 587-611, 1977.

Turcotte, D. L., and J. L. Ahern, A porous flow model for magma migration in the asthenosphere, J. Geophys. Res., 83, 767-772, 1978.

Waff, H. S., and J. R. Bulau, Fluid distribution in partially molten 'pyrolite' (abstract), Eos Trans. AGU, 58, 535, 1977.

Walker, D., J. Longhi, R. J. Kirkpatrick, and J. F. Hays, Differentiation of an Apollo 12 picrite magma, Proc. Lunar Sci. Conf. 7 th. 1365-1389, 1976.

Walker, D., R. J. Kirkpatrick, and J. F. Hays, Differentiation of a komatiite lava (abstract), Eos Trans. $A G U, 58,527,1977$.

Wetherill, G. W., Solar system sources of meteorites and large meteoroids, Annu. Rev. Earth Planet. Sci., 2, 303-331, 1974.

(Received February 7, 1978;

revised August 4, 1978;

accepted September 7, 1978.) 